\title{
Evaluation of Guiard's Theory of Bimanual Control for Navigation and Selection
}

\author{
Xu Xia, Pourang Irani, and Jing Wang \\ Department of Computer Science, University of Manitoba, Canada \\ \{oliverxx, irani, jingwang\}@es.umanitoba.ca
}

\begin{abstract}
Two-handed interaction is a very common paradigm that is gaining popularity in the fields of medical tele-operation, gaming, and large-scale design. In this paper, we validate Guiard's theory of bimanual control for the tasks of navigation and selection. We present the related literature and the theoretical models that motivate the research, in particular Guiard's theory of bimanual control. Two experiments are designed to verify and establish the relationship between navigation and selection in bimanual interaction based on Guiard's theory. The contributions assist interaction designers in developing adequate tools for bimanual operation.
\end{abstract}

Keywords: Unimanual, Bimanual, Navigation, Selection, Dominant Hand, Non-dominant Hand, Guiard's model.

\section{Introduction}

Humans use both hands frequently to perform everyday actions. We naturally use our hands to perform tasks such as picking up an item, washing dishes or in more precise tasks such as hammering a nail to the wall. Typically, our manual operations can be divided into two types: unimaual (one-handed) and bimanual (two-handed). The bimanual operations can be further categorized into symmetric, where both hands perform similar tasks and have the same level of importance; and asymmetric, in which the two hands have different roles at the same time. In bimanual situations, people tend to use one hand for fine operations while the other hand provides a rough guide for the first hand [6]. Researchers have termed these two hands as Dominant Hand (DH) and Non-Dominant Hand (NDH). To a right-handed person, the DH refers to their right hand, and the NDH refers to their left hand.

As a natural way of functioning, two-handed interactions take place in everyday tasks, and with minimal training. In addition, using two hands can reduce the task switching time in one-handed operations. Generally, bimanual interaction, with regards to computing related navigation and selection, is designed to have two input devices and two corresponding cursors. However, people are limited to using their dominant hand to operate the computer in current interfaces. In this paper, we intend to investigate the efficiency of applying theories of bimanual control to tasks that involve navigation in a virtual environment and selection of objects. 


\section{Background and Related Work}

Many studies have examined the nature of bimanual operation and have compared the efficiency between unimanual and bimanual operations, or between symmetric and asymmetric bimanual interactions. Based on a number of theories, researchers have also introduced new bimanual interaction techniques. We present a survey of the literature pertinent to bimanual interaction and the literature that is related to the research proposed here. In this section, we describe the major results that relate to the work carried out here.

\subsection{Bimanual Versus Unimanual Operation}

A number of experiments have been carried out to compare input efficiency between two-handed and one-handed interactions. Similarly, with bimanual interaction, several results describe the nature of symmetric and asymmetric tasks. In one classic study, Buxton and Myers [3] compared the distribution and efficiency of labour with unimanual to bimanual interactions. The participants in their study were grouped into experts and novices. In the first experiment, all the subjects were provided with a graphical drawing interface. Their task consisted of positioning a square in a target place, and to scale it to an expected size. The participants did the experiment with one hand and with two hands. The results of this experiment showed that subjects perform better when they use both hands for simultaneously positioning and scaling an object. In the second experiment, the subjects were required to scroll a word processing document until they found a target word. Buxton and Myers found that, both experts and novices improved in efficiency after changing from one hand to two hands. Furthermore, their results show that the improvement is better for novices than experts. The conclusion of their study suggests that two-handed interaction, for the specific tasks, were more efficient than one-handed operation.

Kabbash et al. [9] examined a one-handed technique and three different types of two-handed techniques. In their experiments, the subjects selected a color from a movable menu and drew lines between displayed vertices. The three bimanual techniques were: i) each hand controls a different cursor with same functionality; ii) the left hand is only responsible for moving the menu, while the right hand is responsible for all the other functions; iii) uses a technique called Toolglass [2], where the colour selection menu is transparent, so that the users can see through the menu. They captured the amount of visual diversion, motor operation and the time for completing the tasks. Their results show that, Toolglass has the least number of motor operations. In addition, only the Toolglass technique out-performs the unimanual technique, while the other two techniques take more time than the unimanual operation. Kabbash et al. concluded that, the method in which the bimanual technique is designed is critical to its efficiency, and "if designed improperly, two hands can be worse than one" [9].

Leganchuk et al. [12] compared two bimanual techniques with traditional unimanual approach. In their research, two experiments were carried out. In the first experiment, the subjects were required to position and resize either an ellipse or a rectangle to minimally cover a given figure in one of six predefined shapes. This experiment compared the traditional unimanual technique (U), a symmetric bimanual 
technique (SB), and a bimanual Toolglass technique (BT). Their results show that, the bimanual techniques outperform the unimanual technique by $17 \%$, while there was no significant difference between the two bimanual techniques. In the second experiment, the users were able to practice before starting the experiment. This time, only U and BT were compared. Their results show that BT outperformed U by $39 \%$. Leganchuk et al. concluded that, cognitive ability is important for performance results, and the mental representation of an ongoing action is important for bimanual interaction performance [12].

In a follow-up to Leganchuk et al. [12], Owen et al. [14] proposed that because the two hands would provide more feedback, manipulation capability, and help to evaluate the data, using both hands are more expressive than using one hand. They investigated the time of completing a unimanual operation and that of completing two bimanual operations with an integrated device for both hands or two separated input devices for each hand. The task in their experiments consisted of manipulating a curve to match a given curve. The authors hypothesized that the one hand task would take longer to complete than the two-hands completion time. They conjectured that part of the overhead would result from a certain amount of cognitive effort. Their results show that the two-handed conditions were approximately $40 \%$ faster than the one-handed conditions. When the task is more complicated, both hands are more efficient. In this study, Owen et al. [14] emphasized integrating bimanual interaction in one input device. However, there is no evidence that shows that an integrated device will outperform a non-integrated setup.

Latulipe et al. [10, 11] compared the efficiency between unimanual (UNI), symmetric bimanual (SYM) and asymmetric bimanual (ASYM) actions using a onemouse interface for unimanual and two-mice interface for bimanual. In their experiments, the users are required to perform an image rotation and scale task. The researchers measured the completion time of performing a task; the response time after the image was shown until the mouse starts to move; the accumulative switch time of the period between the change from one mouse to the other; and the movement time which is the completion time minus the other two. Their results show that the mean completion time of SYM is $87 \%$ faster than UNI, and ASYM is $42 \%$ faster than UNI. Latulipe et al. [11] concluded that, asymmetric bimanual outperforms unimanual actions, while symmetric bimanual technique is the best among the three designs.

In another study, Hinckley et al. [7, 8] designed a task in which the subjects were asked to align virtual objects using one hand and two hands. They provided two tools to control two separate virtual objects. The object would move and rotate according to the operation allowed by the tool. Users could only pickup one tool at a time for the unimanual situation; and would pickup both tools in the bimanual condition. The degree of angle separation between the two objects and the distance between the two objects were recorded. The results show that when subjects use both hands they perform the task more accurately than using one hand only.

The studies above examined the benefits of bimanual interaction in comparison to unimanual operation. The results generally indicate that bimanual interaction, based on a given task, can outperform unimanual interaction. None of the studies, to the best of my knowledge, have investigated the distribution of labour between both hands for the tasks of navigation and selection. In particular, the central question in this study 
inquires as to whether it is better to perform navigation tasks with the NDH and the task of selection with the $\mathrm{DH}$, or the opposite. To resolve this question we first provide a description of Guiard's Kinematic Chain model that motivates my investigation.

\subsection{Guiard's Kinematics Chain Model}

Many bimanual interactive designs have been proposed for various industrial or realworld applications $[4,5,8,9,10,11]$. However, most studies show that, bimanual interaction can be designed in different ways. In particular, the designers are faced with the question of how to distribute the tasks between both hands. To determine the method in which to split the tasks between the left and right hands, it is important to first determine the role of each hand, and to distinguish the tasks that each hand is better at.

To get an answer to the question of the role of each hand, and how to distribute the various tasks between the two hands, we turn to Guiard's theory of bimanual interaction, which is also referred to as Guiard's kinematic chain model. Guiard [6, 13] developed a model to demonstrate the relationship between the roles of both hands in a bimanual application. He defines human hands as two motors as they can make movements, regardless of the internal mechanism of the motion. The movement of such a motor is described in Figure 1. The motor is controlled by an information processing system (IPS), which is analogous to the human brain when the motor represents a human hand. A reference position (RP) generates an input to the motor, and the output of the motor produces a variable position (VP).

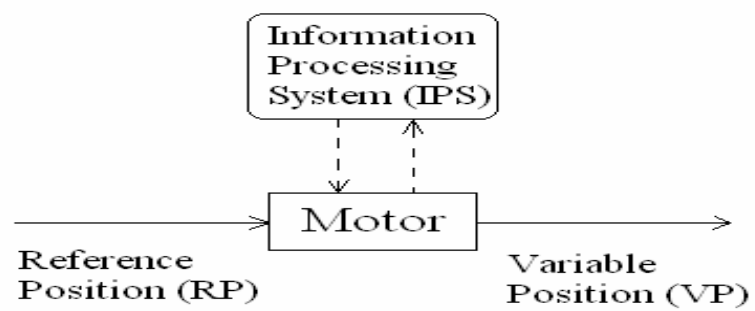

Fig. 1. Representation of a typical motor [8]

Guiard first identified three categories of bimanual actions: orthogonal, where the task of each hand are not related; parallel, where the two hands perform the same task to achieve the same goal; and serial, where the output of one hand is the input of the other hand. In contrast to the first two conditions, the third type of interaction is more natural. Therefore, to take advantage of bimanual interaction, it is best that two hands perform different tasks. This generally often leads to a serial method of processing such that the output from one hand is the input of the other hand. This serialized model is called the kinematic chain model or Guiard's model of bimanual control [6]. In Guiard's model, the non-dominant hand (NDH) acts before the dominant hand (DH), and typically performs a coarse action. The NDH also provides a frame or reference to the $\mathrm{DH}$. The $\mathrm{DH}$ then performs a finer action, which requires the most 
significant cognitive effort from the user. This relation is depicted in Figure 2. The RP for the non-dominant hand (NDH) is the input to the NDH motor. After the movement of the NDH motor, the NDH produces a VP, which together with the RP of the dominant hand $(\mathrm{DH})$ becomes the input of the $\mathrm{DH}$ motor. The motion of the $\mathrm{DH}$ will then generate a VP for the DH. This chain may contain many motors in a serial manner, and the VP for the DH can then become part of the input for the next NDH motor. According to Guiard's model, the chain should always start from the non-dominant hand motor (NDH), and usually end at the dominant hand motor (DH).

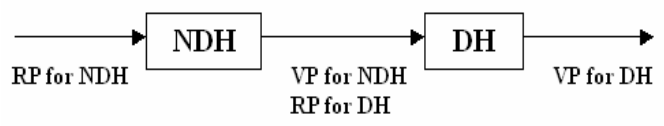

Fig. 2. Guiard's kinematic chain model [8]

Guiard's model is purely descriptive and is summarized in Table 1 below. The actions in this model can be explained by means of a drawing application designed to take advantage of both hands to draw images. The painter is given a template and a pencil. The template will be used by the non-dominant hand (NDH) and the pencil is manipulated by the dominant hand (DH). For simplicity, let us assume that the painter is right-handed and so the $\mathrm{NDH}$ is the left hand, and the $\mathrm{DH}$ is the right hand. To draw figures, the painter will first take the template in the left hand. The template is moved in the drawing space until the painter has a good place to start. In this way the left-hand is setting the spatial frame of reference for the right hand. In handling the template the painter will typically perform coarse movements. Additionally, in this way the movement of the left hand leads the dominant hand, or right hand. Once the position of the template is fixed, the painter works within the established frame of reference set by the left-hand. In this way it has followed the left hand. Finally, to get an image the painter has to perform fine movements. This set of actions is properly encapsulated in Guiard's theory of bimanual control.

Table 1. The roles of both hands in Guiard's Model [4]

\begin{tabular}{|l|ll|}
\hline \multicolumn{1}{|c|}{ Hand } & & \multicolumn{1}{c|}{ Role and Action } \\
\hline Non-Dominant & - & Leads the dominant hand \\
(NDH) & - & Sets the spatial frame of reference for the dominant hand \\
& - & Performs coarse movements \\
\hline Dominant (DH) & - & Follows the non-dominant hand \\
& - & Works within the established frame of reference set by the \\
& & non-dominant hand \\
& - & Performs fine movements \\
\hline
\end{tabular}

As described above a significant number of studies have investigated the applicability or effectiveness of Guiard's theory for bimanual control. The results vary with the different interface designs, the different experimental conditions, tasks, and design. One area that has not been investigated is the use of bimanual interaction for navigation and selection tasks. Navigation and selection are common tasks that are 
carried out in a variety of applications. Navigation is primarily significant in virtual environments, in 3D interfaces, and in applications that requires visual searching and browsing. Selection is common in most graphical user interfaces. More specifically, bimanual interaction with navigation and selection has been applied to medical telelearning (the trainee will navigate in a virtual environment using one hand and perform selections with another), in tele-surgery (the surgeon will navigate in the environment the represents the patients body and use the other hand to perform precise stitching or picking), and in video games.

In this paper we are concerned with finding out how we assign the different tasks of navigation and selection to the different hand. More specifically, do we assign the task of navigation to the $\mathrm{DH}$ and selection to the NDH or do we do the reverse? The objective of this study is to verify the implication of Guiard's theory of bimanual interaction for the tasks of navigation and selection.

\section{Study}

To determine how to assign tasks to the non-dominant hand and to the dominant hand we first describe the nature of navigation and selection. Bimanual navigation and selection requires continuous and asymmetric behavior. Navigation may not require very precise movements, but rather it can be coarse, and it typically sets the frame of reference. Selection is precise, requires attention to detail, and will typically be working in a frame of reference that has already been instantiated. Based on Guiard's theory of bimanual control and the results of prior work, we expect that users will perform better when navigation is relegated to the non-dominant hand and selection to the dominant hand (primary hypothesis). In the following section we describe the experimental set-up that will be designed to test the hypothesis.

\subsection{Design}

To verify the hypothesis we conducted two separate experiments. In the first experiment, the users navigated and selected objects in an environment where the targets are static. In the second experiment, the targets are dynamic. In both experiments, we test the effect of using the dominant and non-dominant hand as suggested by Guiard's model

Experiment 1: Static objects. The objective of this experiment is to validate Guiard's theory for the tasks of selection and navigation with static targets.

Materials. The experiment will be implemented on an Intel Pentium 4 CPU and 19" $1280 \times 1024$ resolution display monitor. The operating system will be Windows XP. The input devices for navigation and selection are two Logitech Extreme 3D joysticks.

Implementation. The implementation is carried out using the Microsoft .NET C\# environment. 
Description of the task. To validate Guiard's theory of bimanual control we designed a task that necessitates navigation and selection. The system presents the users a maze in which they navigate and select specific objects. Both navigation and selection is performed using two joysticks. The task is performed under different conditions as described below. The following figure depicts a possible scenario.

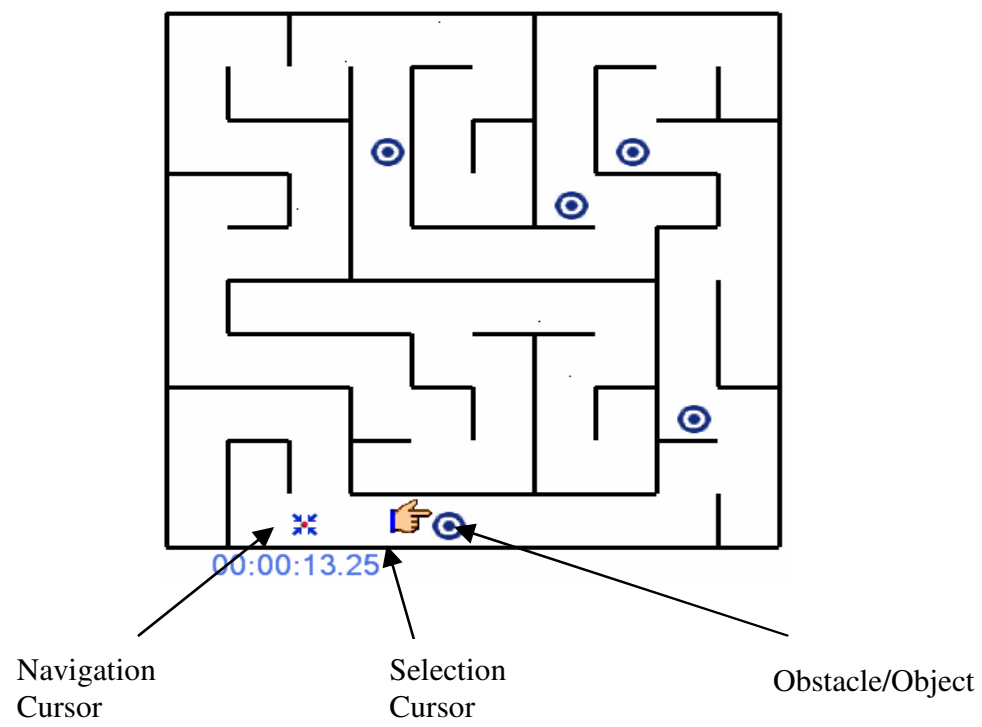

Fig. 3. Interface of the maze for the experiment

Both joysticks' cursors are positioned at the start location which will be the left bottom of the maze at the beginning of each trial. The objective is to exit from the maze by navigating throughout the maze removing obstructions by selecting them. With the navigation joystick the user starts to navigate along the route. The participant has to eliminate every object that appears on the route using both joysticks. This task is representative of tasks that require some form of navigation toward the objects and then some selection. We record the time to complete the task.

Design. The experiment uses a $2 \times 2 \times 2$ within-subject design. The main factors for this experiment are task division ( 2 levels) and maze complexity which is composed of number of turns (few and many) and the width of paths (small and large). Each of these factors is described in some detail below.

Task Division. To verify the application of Guiard's theory for bimanual operation, the experiment assigns two types of task division to the dominant hand and nondominant hand. In the first condition the navigation joystick is set to the $\mathrm{DH}$ and the selection joystick to the NDH. In the second condition we reverse the control of each joystick. 
Number of turns. One level of complexity is the different number of turns along the route from the start point to the end point. There are two levels of turns for this experiment with 10 and 20 turns for each trial.

Width of paths. To add additional complexity, the experiment varies the different widths of paths from the start to the end. This will be set according to the Steering Law [1]. This factor has two levels, one of 5 pixels and the other of 10 pixels wide.

Experiment 2: Dynamic Objects. Experiment 2 is identical to Experiment 1 with the difference that objects are dynamic and the user has to navigate toward the dynamic objects and select them. The methodology is similar and so we report the results of both experiments together.

\section{Results and Analysis}

For each trial the system records the time it takes the user to navigate and select objects in the entire maze. We analyze the results by means of a $2 \times 2 \times 2$ (Task Division $\times$ Number of Turns $\times$ Path Width) repeated measures analysis of variance (ANOVA) with Task Division, Number of Turns, and Path Width serving as repeated measures. The experiment collects the completion time for each trial. Through both experiments, we expect to validate Guiard's theory. Namely, the results will validate whether Guiard's theory applies to the tasks of navigation and selection in virtual environments.

\subsection{Results for Static Experiment}

In experiment 1 , we obtained the following results:

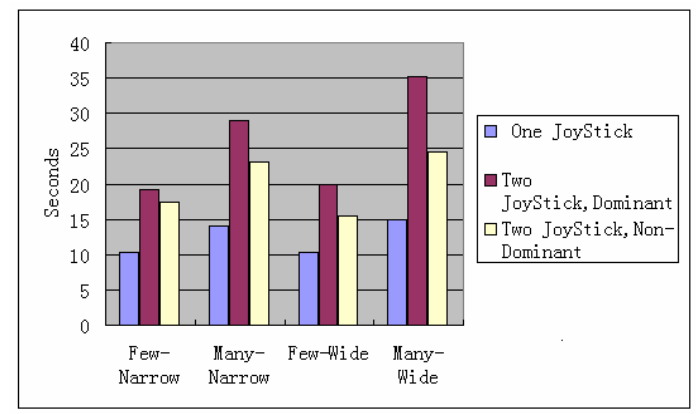

Fig. 4. Chart of experimental results

The preliminary results (figure 4) with twelve participants show that navigation and selection can be assigned to the two different hands based on Guiard's model, i.e. navigation can be relegated to the non-dominant hand and selection to the dominant hand. 
The results are consistent across all participants. In summary we see that:

1. Unimanual is faster than bimanual operation.

2. In bimanual experiments, non-dominant hand (NDH) for navigation is faster than dominant hand $(\mathrm{DH})$ for navigation.

\subsection{Results for Dynamic Experiments}

Similar to static experiments, the dynamic experiments produced consistent results that show same features as static experiments, namely:

1. Unimanual is faster than bimanual operation.

2. In the bimanual condition, non-dominant hand (NDH) for navigation is faster than dominant hand (DH) for navigation.

The overall results for static and dynamic experiments are similar and so we combine the discussion for these two experiments.

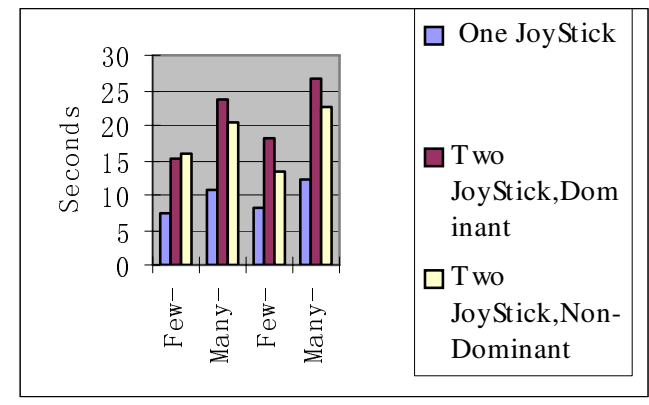

Fig. 5. Chart of experimental results

\subsection{Analysis}

In our experiments we found that unimanual operation performs better than when navigation is given to both the dominant hand and the non-dominant hand in bimanual experiments. This result seems contrary to the Guiard's Kinematics Chain Model. However, based on investigation of testers' feedback and extensive study, we find the task design of this experiment is not very typical for bimanual operation. In general the design of navigation and selection may have been improved if the operation was performed in a symmetric manner. In this case, our task did not require symmetric operation and for that reason it is not clear whether there are any benefits to bimanual operation under such conditions.

In both experiments, the results show that navigation to the non-dominant hand outperforms navigation in the dominant hand. One reason for this is that users found it difficult to perform the selection operation. In this case, selection required that the user follow the navigation hand and then perform a precise selection of the target. This can be difficult given the complexity of the space users are required to navigate and select objects. 


\section{Conclusions and Contributions}

According to the analyses above, we conclude that:

- bimanual interaction does not perform better than unimanual interaction for tasks that are asymmetric;

- definition of dominant and non-dominant hand depends on the workload of the tasks assigned to each hand.

Buxton and Myers's contribution [7] is that they tested bimanual interaction in very simple navigation and selection environments using the mouse, which is only 1DOF navigation device. This device is limited in terms of navigation for many tasks, such as those seen in games. In this research, we tested bimanual interaction under real-time navigation and selection environment using two joystick input device. This work has not been carried out previously. Furthermore, there is very little work in bimanual interaction with a $2 \mathrm{D}$ navigation device such as joysticks.

\section{References}

[1] Accot, J., Zhai, S.: Beyond Fitt's Law: Models for Trajectory-Based HCI Tasks. In: Proc. of CHI'97, pp. 295-302 (1997)

[2] Bier, E.A., Stone, M.C., Poer, K., Buxton, W., DeRose, T.: In: Kajiya, J.T.: Toolglass and Magic Lenses: The see-through interface. In: Proc. of SIGGRAPH, pp. 73-80 (1993)

[3] Buxton, W., Myers, B.A.: Study in Two-handed Input. In: Proc. of CHI'86, pp. 321-326 (1986)

[4] Carroll, J.M.: HCI Models, Theories, and Frameworks toward a Multidisciplinary Science. Morgan Kaufmann Publishers, San Francisco (2003)

[5] Fitzmaurice, G., Baudel, t., Kurtenbach, G., Buxton, B.: The Design of a GUI Paradigm based on Tablets, two-hands, and Transparency. In: Proc. CHI'97, pp. 35-42 (1997)

[6] Guiard, Y.: Asymmetric Division of Labor in Human Skilled Bimanual Action: The Kinematic Chain as a Mode. The Journal of Motor Behaviour 19(4), 486-517 (1987)

[7] Hinckley, K., Pausch, R., Proffitt, D.: Attention and Visual Feedback: The Bimanual Frame of Reference. In: Proc. SIGGRAPH 1997, pp. 121-126 (1997)

[8] Hinckley, K., Pausch, R., Proffitt, D., Patten, J., Kassell, N.: Cooperative Bimanual Action. In: Proc. CHI' 97, pp. 27-23 (1997)

[9] Kabbash, P., Buxton, W., Sellen, A.: Two-handed input in a compound task. In: Proc. CHI'94, pp. 417-423 (1994)

[10] Latulipe, C., Kaplan, C.S., Clarke, C.: Bimanual and Unimanual Image Alignment: An evaluation of Mouse-Based Techniques. In: Proc. on UIST'05, pp. 123-131 (2005)

[11] Latulipe, C., Laplan, C.S., Clarke, C.: Mouse-based Rotation and Translation. In: Proc. of HCI'05, pp. 63-67 (2005)

[12] Leganchuk, A., Zhai, S., Buxton, W.: Manual and cognitive benefits of two-handed input an experimental study. ACM Transactions on Computer-Human Interaction 5(4), 326359 (1998)

[13] MacKenzie, I.S., Guiard, Y.: The Two-handed Desktop Interface: Are we there yet? In: Proc. CHI'01, pp. 351-352 (2001)

[14] Owen, R., Kurtenbach, G., Firzmaurice, G., Baudel, T., Buxton, B.: When it gets more difficult, use both hands - exploring bimanual curve manipulation. In: Proc. of Graphics Interface 2005, pp. 17-24 (2005) 